\title{
Exacerbación de asma infantil en Unidad de Paciente Crítico: Caracterización clínica
}

\author{
ANA MARÍA HERRERA G.*, ANA MARÍA ESCOBAR C.*, MÓNICA SAAVEDRA B.*, \\ MARÍA EUGENIA MEDINA R.**, EDUARDO SALGADO B.***, \\ TAMARA PALAVECINO B.*** y ANA MARÍA KUTZ E.****
}

Asthma exacerbation in a Pediatric Intensive Care Unit: clinical features

Introduction: Some asthmatic children present severe exacerbations, demanding hospitalization in the Pediatric Intensive Care Unit (PICU). On a national Chilean scale, there is no published data about these patients. The objective of this study was to evaluate the clinical features of children with asthmatic exacerbations requiring PICU management at Roberto del Rio Children's Hospital in Santiago de Chile. Patients and Method: Retrospective and descriptive study in children above 4 years of age discharged from PICU with diagnosis of asthmatic exacerbation during a three years period. Results: 67 children required treatment in PICU. The average of age was 7 years old, while the average of hospital stay was 4 days. Non invasive ventilation (NIV) was used in 59 patients. Only 27 patients had previous diagnosis of asthma, with good treatment adherence only in 7 of them. Conclusions: Before being hospitalized, only $40 \%$ of the asthmatic patients were diagnosed as asthmatic and most of them had a bad treatment compliance. NIV was used in a remarkably high percentage of these patients.

Key words: Asthma attack, near fatal asthma, asthma crisis. Pediatric intensive care unit.

\section{Resumen}

Introducción: Algunos niños asmáticos presentan exacerbaciones que motivan su ingreso a Unidad de Paciente Crítico (UPC). A nivel nacional no existen publicaciones al respecto. El objetivo del estudio fue conocer las características clínicas de los niños con crisis asmáticas que requieren manejo en UPC en el Hospital Roberto del Río. Pacientes y Método: Estudio retrospectivo, descriptivo en mayores de 4 años, egresados de UPC con diagnóstico de crisis de asma en un periodo de tres años. Resultados: 67 niños requirieron manejo en UPC. El promedio de edad fue 7 años y el de hospitalización 4 dias. En 59 pacientes se utilizó Ventilación Mecánica No Invasiva (VMNI). Del total de pacientes sólo 27 tenía diagnóstico previo de asma, observándose buena adherencia al tratamiento sólo en 7. Conclusiones: Sólo el 40\% de los pacientes asmáticos tenían el diagnóstico de asma antes de la hospitalización y la mayoría de ellos presentaba una pobre adherencia al tratamiento. En el manejo destaca un alto porcentaje de uso de VMNI.

Palabras clave: Asma grave, exacerbación severa, crisis de asma. Unidad de cuidado intensivo pediátrico.

\footnotetext{
* Pediatra Especialista en Enfermedades Respiratorias Infantiles, Departamento de Pediatría Norte, Facultad de Medicina, Universidad de Chile. Hospital Roberto del Río.

** Tecnólogo Médico. Hospital Roberto del Río.

*** Interno Medicina, Universidad de Chile.

**** Interno Medicina, Universidad del Desarrollo.
} 


\section{Introducción}

El asma bronquial es la enfermedad crónica más frecuente en la infancia ${ }^{1}$. La prevalencia de asma ha aumentado en distintas partes del mundo, especialmente en el grupo menor de 12 años ${ }^{1}$. La prevalencia de asma pediátrico en nuestro país varía entre 7,3 y $16,5 \%{ }^{2,3}$. A pesar de que en Chile el sistema de salud asegura que los pacientes asmáticos reciban tratamiento, vemos que inevitablemente se siguen hospitalizando niños con esta patología, algunos de los cuales evolucionan gravemente requiriendo manejo en unidad de pacientes críticos (UPC). Estas exacerbaciones ponen en riesgo la vida del paciente, significando además un importante gasto en salud. En la literatura nacional no hay publicaciones acerca de las características de los niños asmáticos con exacerbaciones severas que requieren manejo en UPC, por lo que el objetivo de este estudio fue conocer las características clínicas de estos pacientes en el Hospital Roberto del Río.

\section{Pacientes y Método}

Se realizó un estudio retrospectivo, descriptivo, en niños mayores de 4 años con exacerbación de asma, que fueron manejados en UPC del Hospital Roberto del Río entre Enero de 2005 y Diciembre de 2007. Decidimos incluir sólo a los mayores de 4 años para tener mayor seguridad con respecto al diagnóstico de asma. No se incluyó a pacientes con crisis obstructivas que eran portadores de otras patologías crónicas como fibrosis quística, displasia broncopulmonar, daño pulmonar crónico y cardiopatías. Lo anterior para poder analizar los datos de los pacientes puramente asmáticos. Se revisó en forma manual el libro de egresos de la UPC obteniéndose las fichas médicas de los niños mayores de 4 años con diagnóstico de crisis asmática. De las fichas clínicas se extrajeron distintos datos relacionados con el paciente y sus antecedentes (edad, género, diagnóstico previo de asma, tratamiento de mantención, presencia de atopía, test cutáneo, espirometría, test de metacolina) y datos relacionados con la hospitalización (duración, posibles gatillantes, exámenes y tratamiento administrado). Estas distintas variables clínicas fueron tabuladas y analizadas, expresando los resultados en porcentajes y promedios.

\section{Resultados}

Durante los 3 años estudiados, 67 pacientes mayores de 4 años egresaron de UPC con diag- nóstico de crisis asmática, siendo 36 de género femenino. La edad promedio fue de 7 años. El grupo mayoritario correspondió a niños de 4 a 9 años $(83,5 \%)$.

Dentro de los antecedentes previos a la hospitalización destaca que 33/67 (49,2\%) tenía al menos una hospitalización previa por crisis de asma y en 5 de ellos $(7,4 \%)$ al menos una en UPC. Un 40\% (27/67) de los niños tenía diagnóstico establecido de asma (Tabla 1). Todos estos pacientes registraban controles en Policlínico de Broncopulmonar y tenían indicado tratamiento con corticoides inhalados. Sin embargo, en 20 de 27 la asistencia era irregular y presentaban mala adherencia al tratamiento. Cuarenta de los 67 pacientes $(60 \%)$ no tenía diagnóstico previo de asma. Sin embargo, 32 refería el antecedente de obstrucción bronquial previa. En 8/67 pacientes $(11,9 \%)$ la hospitalización en UPC fue la primera manifestación de asma bronquial. Al analizar la presencia de atopía observamos que 28/67 niños $(41,7 \%)$ tenían rinitis alérgica y $21 / 67(31,3 \%)$ dermatitis atópica. Al momento de la revisión de las fichas clínicas $34 / 67$ pacientes $(50,7 \%)$ se habían realizado test cutáneo. Este examen fue positivo al menos a un aeroalergeno en $25 / 34$ niños. El test de metacolina había sido efectuado a $29 / 67$ niños (43,2\%), siendo positivo en $21 / 29$ $(72,4 \%) ; 6$ presentaban hiperreactividad leve, 8 moderada y 7 severa.

Con respecto a la hospitalización, observamos que la duración de la sintomatología previa al ingreso fue menor a 48 horas en 30 pacientes $(44,7 \%)$ y menor a 24 horas en $12(17,9 \%)$. El promedio de días en UPC fue de 4 (1-10). Con respecto a los posibles gatillantes se realizó estudio de virus respiratorios mediante inmunofluorescencia a $35 / 67$ pacientes $(52 \%)$ resultando positiva en 9/35 (26\%): 4 virus respiratorio sincicial, 3 virus parainfluenza y 2 virus influenza. Se sospechó la presencia de Mycoplasma pneumoniae en 25/67 niños (37,3\%). Se realizó IgM

Tabla 1. Antecedentes de asma antes de la hospitalización

\begin{tabular}{|lcc|}
\hline & n & \% \\
\hline Diagnóstico de asma & 27 & 40,2 \\
\hline $\begin{array}{l}\text { Sin diagnóstico de asma pero con } \\
\text { antecedentes de obstrucción bron- } \\
\text { quial previa }\end{array}$ & 32 & 47,7 \\
$\begin{array}{l}\text { Primer episodio de obstrucción } \\
\text { bronquial }\end{array}$ & 8 & 11,9 \\
\hline
\end{tabular}


Tabla 2. Tratamiento recibido en UPC

\begin{tabular}{|lcc|}
\hline & n & $\%$ \\
Oxígeno & 67 & 100 \\
Salbutamol nebulizado intermitente & 67 & 100 \\
Hidrocortisona endovenosa & 67 & 100 \\
Aminofilina endovenosa & 40 & 59,7 \\
Macrólidos & 25 & 37,3 \\
Otros Antibióticos & 7 & 10,4 \\
VMNI & 59 & 88 \\
VMI & 2 & 2,98 \\
\hline
\end{tabular}

para Mycoplasma pneumoniae a 16 de ellos, confirmándose su presencia en 4 . En $100 \%$ de los niños el patrón radiológico fue infiltrado intersticial de distinta cuantía e hiperinsuflación. Las complicaciones más frecuentes fueron la aparición de atelectasias en 33/67, seguido de bronconeumonia en 4/67 y neumomediastino en 2/67. La totalidad de los niños fue tratado con oxígeno, salbutamol nebulizado e hidrocortisona endovenosa (Tabla 2). En 59/67 pacientes se utilizó VMNI, siendo 4 (1-8) el promedio de días de apoyo ventilatorio. En sólo 2/67 pacientes (2,9\%) se utilizó ventilación mecánica invasiva (VMI). Uno requirió además ventilación de alta frecuencia. No hubo mortalidad en los niños estudiados.

\section{Discusión}

Nuestro estudio tiene la debilidad de ser retrospectivo, descriptivo y de revisión de fichas clínicas, razón por la cual no contamos con la totalidad de los datos para cada uno de los pacientes, como estudio de agentes infecciosos y estudio de función pulmonar entre otros. Sin embargo, tiene la fortaleza de presentar por primera vez datos acerca de las características clínicas de las exacerbaciones de asma que requieren tratamiento en UPC en niños chilenos. Según el Center for Disease Control and Prevention (CDC) de Estados Unidos la tasa de hospitalización en niños asmáticos es de 27/10.000 habitantes ${ }^{4}$, reportándose que un $2 \%$ requiere manejo en $\mathrm{UPC}^{5}$. Para estimar la proporción de los asmáticos hospitalizados que requirieron manejo en UPC en el período de estudio, solicitamos al Servicio de
Estadística el número total de niños mayores de 4 años egresados con diagnóstico de asma entre Enero de 2005 y Diciembre de 2007. Observamos que de un total de 399 egresos hospitalarios por asma 67 requirió UPC (16,7\%). Este mayor porcentaje obtenido en relación a lo reportado en la literatura podría explicarse por diferencias en los criterios de ingreso a UPC. Con respecto a la edad observamos que más del $80 \%$ de nuestros niños eran menores de 10 años, lo que concuerda con otros datos publicados en que se evidencia una marcada disminución de la tasa de hospitalización en los niños mayores de 10 años ${ }^{6}$.

En relación a los antecedentes clínicos de nuestros niños observamos que cerca de la mitad registraba al menos una hospitalización previa por crisis de asma, resultado similar a otras publicaciones que reportan un 42 a $77 \% \%^{7}$. Del total de niños 7,4\% había sido hospitalizado previamente en UPC, cifra comparativamente menor al 11 y $24 \%$ reportado por otras series ${ }^{5,8}$. La mayoría de nuestros pacientes con diagnóstico establecido de asma, registraban asistencia irregular a controles con especialista y mala adherencia al tratamiento indicado. Creemos que estos datos son de gran importancia en cuanto a morbilidad en asma bronquial ya que la falta de adherencia es un factor potencialmente modificable a través de la educación del paciente y sus familias, instancia deficitaria en la mayoría de los servicios públicos. Otro aspecto que llama fuertemente la atención es la falta de diagnóstico de asma en nuestros niños. De los 40 pacientes sin diagnóstico de asma, $80 \%$ refería cuadros de obstrucción bronquial a repetición. La tardanza en el diagnóstico incide en la falta de tratamiento oportuno, predisponiendo al paciente a tener exacerbaciones potencialmente graves.

La presencia de atopía fue una característica importante en los niños estudiados. Un $41,7 \%$ tenía rinitis alérgica y un tercio dermatitis atópica, resultados similares a los descritos por Vicuña et $\mathrm{al}^{9}$. Al momento del estudio la mitad de los pacientes tenía test cutáneo. Este fue positivo al menos a un aeroalergeno en $73,5 \%$ de los casos, cifra similar al $77 \%$ reportado por otras series ${ }^{8}$. El resultado del test de metacolina de los 29 niños que tenían este examen refleja que independiente del grado de hiperreactividad bronquial, el paciente puede experimentar una exacerbación severa. Esto está indicando que la función pulmonar puede no reflejar la verdadera condición del paciente, remarcando la importancia del concepto actual de control del asma.

Los principales gatillantes de exacerbación de asma son las infecciones virales, las cuales están 
presentes en un 80 a $85 \%$ de los casos, siendo rinovirus el agente más prevalente ${ }^{10}$. Observamos que el estudio virológico no se realizó de rutina a todos los pacientes, por lo que la detección fue baja. Además nuestro hospital no dispone de técnicas para estudio de rinovirus. Otros agentes infecciosos asociados a exacerbación de asma son las bacterias atípicas como Mycoplasma pneumoniae, encontrándose en porcentajes que varían entre un 7,5 a $20 \% \%^{8,9,11}$. En nuestra serie se detectó en $5,9 \%$ de los casos, debiéndose consignar que no se pudo realizar el estudio a todos los pacientes en que se sospechó este agente infeccioso por problemas de costo.

Con respecto al manejo observamos que casi $60 \%$ de los niños recibió aminofilina endovenosa. Esta se agregó al uso de salbutamol y corticoides sistémicos cuando había una respuesta parcial al tratamiento. En estos pacientes se consigna un efecto beneficioso en el puntaje clínico. Al revisar la literatura se puede observar que hay diferencias significativas en cuanto a la frecuencia de uso de este medicamento en UPC dependiendo del centro. Es así como se describe su uso en 0 a $24 \%$ de niños manejados en UPC sin ventilación mecánica y en 0,6 a $59 \%$ de los que sí la reciben ${ }^{12}$. La utilidad del uso de aminofilina es controvertida. En un estudio randomizado, doble ciego, realizado en 163 niños se vió que el uso de aminofilina mejoraba la saturación de oxígeno y la función pulmonar ${ }^{13}$. En otro trabajo caso control efectuado en 47 niños se observó que el uso de aminofilina agregado al de salbutamol, bromuro de ipatropio y metilprednisolona proveía un efecto beneficioso en el puntaje clínico $^{14}$. En ninguno de los estudios mencionados se demuestra una disminución de los días de hospitalización. Finalmente en un meta-análisis que incluyó a 380 niños se concluye que el uso de aminofilina mejora la función pulmonar a las 6 horas de uso, sin una reducción aparente de síntomas y de días de hospitalización, observándose un aumento del riesgo de vómitos ${ }^{15}$. Aparte del tratamiento farmacológico una alta proporción de nuestros pacientes recibió VMNI (88\%). Este porcentaje es muy superior al 4 a $20 \%$ publicado por otras series ${ }^{7,8}$. La UPC del Hospital Roberto del Río ha sido pionera en el uso de la VMNI en Chile, teniendo gran experiencia en su uso. Probablemente la alta proporción de uso de VMNI en comparación con lo reportado en la literatura se debe a un ingreso precoz de los pacientes en un intento por disminuir el esfuerzo respiratorio evitando la fatiga de los músculos respiratorios y la necesidad de intubación ${ }^{16}$. El uso de VMI en nuestra serie fue de $2,9 \%$. De acuerdo a las distintas publicaciones su uso es muy variable dependiendo del centro estudiado, con cifras que van desde 2,8 hasta $33 \%$,

\section{Bibliografía}

1.- WERNER H. Status asthmaticus in children. Chest. 2001; 119: 1913-29.

2.- MALLOL J, CORTEZ E, AMARALES L, SÁNCHEZ I, CALVO M, SOTO S, et al. Prevalencia del asma en escolares chilenos. Estudio descriptivo de 24470 niños. ISAAC-Chile. Rev Med Chile 2000; 128: 279-85.

3.- MALLOL J, AGUIRRE V, AGUILAR P, CALVO M, AMARALES L, ARELLANO P, et al. Cambios en la prevalencia de asma en escolares chilenos entre 1994 y 2002. Rev Med Chile 2007; 135: 580-6.

4.- GRINESKI S. Characterizing children's asthma hospitalizations on the Texas-Mexico border. Journal of asthma 2007; 44: 783-7.

5.- STEIN R, CANNY G, BOHN D, REISMAN J, LEVISON H. Severe acute asthma in a pediatric intensive care unit: six years experience. Pediatrics 1989; 83: 1023-8.

6.- GETAHUN D, DEMISSIE K, RHOADS G. Recent trends in asthma hospitalization and mortality in the United States. Journal of asthma 2005; 42: 373-8.

7.- CARROLL C, SCHRAMM C, ZUCKER A. Severe exacerbations in children with mild asthma: characterizing a pediatric phenotype. Journal of asthma 2008; 45 : 513-7.

8.- BELESSIS Y, DIXON S, THOMSEN A, DUFFY B, RAWLINSON W, HENRY R, et al. Risk factors for an intensive care unit admission in children with asthma. Pediatr pulmonol 2004; 37: 201-9.

9.- VICUÑA P, LOZA C, MUÑOZ P, SÁNCHEZ I. Características clínicas de los niños asmáticos hospitalizados en un servicio de pediatría. Rev Chil Ped 2007; 78: 29 34.

10.- SINGH AM, BUSSE W W. Asthma exacerbations 2: Aetiology. Thorax 2006; 61: 809-16.

11.- BISCARDI S, LARROT M, MARC E, MOULIN F, BOUTONNAT-FAUCHER B, HEILBRONNER C, et al. Mycoplasma Pneumoniae and asthma in children. Clin Infect Dis 2004; 38: 1341-6.

12.- BRATTON S, ODETOLA F, MCCOLLEGAN J, CABANA M, LEVY F, KEENAN H. Regional variation in ICU care for pediatric patients with asthma. J Pediatr 2005; 147: 355-61.

13.- YUNG M, SOUTH M. Randomized controlled trial of aminophylline for severe acute asthma. Arch Dis Child 1998:79: 405-10.

14.- REAM R, LOFTIS L, ALBERS G, BECKER B, LYNCH R, MINK R. Efficacy of IV theophylline in children with severe status asthmaticus. Chest 2001; 119: $1480-8$. 
15.- MITRA A, BASSLER D, WATTS K, LASSERSON T, DUCHARME F. Intravenous aminophylline for acute severe asthma in children over two years receiving inhaled bronchodilators. Cochrane Database of systematic review. 1, 2009.
16.- YÁÑEZ L, YUNGE M, EMILFORK M, LAPADULA M, ALCÁNTARA A, FERNÁNDEZ C, et al. A prospective, randomized, controlled trial of noninvasive ventilation in pediatric acute respiratory failure. Pediatr Crit Care Med 2008; 9: 484-9.

Correspondencia a:

Dra. Ana María Herrera Gana

Bellavista 0415. Santiago, Chile.

Fono: 9130255

E-mail amherrerag@yahoo.com 